\title{
Third order problems with nonlocal conditions of integral type
}

\section{Abdelkader Boucherif ${ }^{*}$, Sidi Mohamed Bouguima², Zehour Benbouziane ${ }^{2}$ and Nawal Al-Malki}

\section{"Correspondence:}

aboucher@kfupm.edu.sa

'Department of Mathematics and

Statistics, King Fahd University of

Petroleum and Minerals, P.O. Box

5046, Dhahran, 31261, Saudi Arabia

Full list of author information is

available at the end of the article

\begin{abstract}
We discuss the existence of solutions of nonlinear third order ordinary differential equations with integral boundary conditions. We provide sufficient conditions on the nonlinearity and the functions appearing in the boundary conditions that guarantee the existence of at least one solution to our problem. We rely on the method of lower and upper solutions to generate an iterative technique, which is not necessarily monotone.

MSC: 34B10; 34B11; 34B12; 34B13; 34B14; 34B15

Keywords: third order differential equations; integral boundary conditions; a priori bound on solutions; fixed point theorems; lower and upper solutions; iterative technique
\end{abstract}

\section{Introduction}

The purpose of this paper is to establish the existence of solutions for a class of nonlinear third order ordinary differential equations with integral boundary conditions. More specifically, we consider the following problem:

$$
\begin{aligned}
& u^{\prime \prime \prime}(t)+f\left(t, u(t), u^{\prime}(t), u^{\prime \prime}(t)\right)=0, \quad t \in[0,1], \\
& u(0)=0, \\
& u^{\prime}(0)-a u^{\prime \prime}(0)=\int_{0}^{1} h_{1}\left(u(s), u^{\prime}(s)\right) d s, \\
& u^{\prime}(1)=\int_{0}^{1} h_{2}\left(u(s), u^{\prime}(s)\right) d s,
\end{aligned}
$$

where $f:[0,1] \times \mathbb{R}^{3} \rightarrow \mathbb{R}, h_{1}, h_{2}: \mathbb{R}^{2} \rightarrow \mathbb{R}$ are continuous functions, and $a$ is a nonnegative real number. Several papers have been devoted to the study of third order differential equations with two-point and three-point boundary conditions. See [1-3], and [4] for references. Problems with integral boundary conditions have been used in the description of many phenomena in the applied sciences. We refer the interested reader to [5] and the references therein. Very few papers have dealt with nonlocal conditions for third order differential equations. We can mention [6, 7] and [8]. For higher order differential equations with functional boundary conditions the interested reader can consult [9]. In this work we use the method of lower and upper solutions to generate a sequence of modified nonlinear problems, each having a unique solution; in this way, we obtain a sequence of

(c) 2014 Boucherif et al.; licensee Springer. This is an Open Access article distributed under the terms of the Creative Commons Attribution License (http://creativecommons.org/licenses/by/2.0), which permits unrestricted use, distribution, and reproduction in any medium, provided the original work is properly credited. 
functions, which is uniformly bounded together with their first and second order derivatives. We then extract a subsequence converging uniformly to a solution of our original problem (1)-(4). Contrary to many works in the literature, we develop an iterative technique, which is not necessary monotone. We should point out that our approach is totally different from that of [9].

\section{Preliminaries}

Let $I$ denote the real interval $[0,1] . C(I)$ is the Banach space of real-valued continuous functions on $I$, equipped with the norm $\|u\|_{0}:=\max \{|u(t)| ; t \in I\}$, for $u \in C(I)$. Let $D$ denote the set of all real-valued functions which are three times continuously differentiable on $I$. We define the norm of $u \in D$ by

$$
\|u\|_{D}=\|u\|_{0}+\left\|u^{\prime}\right\|_{0}+\left\|u^{\prime \prime}\right\|_{0}+\left\|u^{\prime \prime \prime}\right\|_{0} .
$$

Let $D_{0}=\{u \in D ; u(0)=0\}$. Then $\left(D_{0},\|\cdot\|_{D}\right)$ is a Banach space.

Definition 1 A solution of problem (1)-(4) is a function $u \in D_{0}$ that satisfies (1) for every $t \in I$ and the conditions (3) and (4).

Definition 2 Let $\alpha, \beta \in D_{0}$ satisfy $\alpha^{\prime}(t) \leq \beta^{\prime}(t)$ for every $t \in I$. We denote by $\left[\alpha^{\prime}, \beta^{\prime}\right]$ the set of all $v \in D_{0}$ such that $\alpha^{\prime}(t) \leq v(t) \leq \beta^{\prime}(t)$ for every $t \in I$.

It is clear that if $u^{\prime} \in\left[\alpha^{\prime}, \beta^{\prime}\right]$ and $u \in D_{0}$, then $u \in[\alpha, \beta]$.

Definition 3 Let $\alpha, \beta \in D_{0}$ satisfy $\alpha^{\prime}(t) \leq \beta^{\prime}(t)$ for every $t \in I$. Let $S(\alpha, \beta)$ denote the set of all functions $u \in D_{0}$ such that $u \in[\alpha, \beta]$ and $u^{\prime} \in\left[\alpha^{\prime}, \beta^{\prime}\right]$.

Remark 1 It is clear that $u \in D_{0}$ and $u^{\prime} \in\left[\alpha^{\prime}, \beta^{\prime}\right]$ imply that $u \in S(\alpha, \beta)$.

Definition 4 Let $\alpha, \beta \in D_{0}$ satisfy $\alpha^{\prime}(t) \leq \beta^{\prime}(t)$ for every $t \in I$. Define the operator $p$ : $D_{0} \rightarrow[\alpha, \beta]$ by

$$
(p u)(t)=\max \{\alpha(t), \min (u(t), \beta(t))\}, \quad \forall t \in I,
$$

and the operator $q: D_{0} \rightarrow\left[\alpha^{\prime}, \beta^{\prime}\right]$ by

$$
(q v)(t)=\max \left\{\alpha^{\prime}(t), \min \left(v(t), \beta^{\prime}(t)\right)\right\}, \quad \forall t \in I .
$$

Remark 2 The operators $p$ and $q$ are continuous and bounded.

\section{Main results}

In this section we state and prove our main results. The first result is of independent interest and plays a key role in the proof of our second result.

Theorem 1 Let $\phi: I \times \mathbb{R} \rightarrow \mathbb{R}$ be continuous, bounded and satisfy the following condition:

$\left(\mathrm{H}_{\phi}\right)\left(\phi\left(t, v_{2}\right)-\phi\left(t, v_{1}\right)\right)\left(v_{2}-v_{1}\right)<0$ for all $v_{1}, v_{2} \in \mathbb{R}$ such that $v_{1} \leq v_{2}$ and $t \in I$. 
Then for any $\delta, \rho$ the boundary value problem

$$
\left\{\begin{array}{l}
-u^{\prime \prime \prime}(t)=\phi\left(t, u^{\prime}(t)\right), \quad t \in I, \\
u^{\prime}(0)-a u^{\prime \prime}(0)=\delta, \\
u^{\prime}(1)=\rho
\end{array}\right.
$$

has a unique solution $u$.

Proof Uniqueness. Suppose that problem (5) has two solutions $x$ and $u$ in $D_{0}$. Put $z=x^{\prime}-u^{\prime}$. Then $z(1)=x^{\prime}(1)-u^{\prime}(1)=0$. We show that $z(0)=0$. Suppose this is not true. Then either $z(0)>0$ or $z(0)<0$. We consider the case $z(0)>0$. From the condition at $t=0$ it follows that $0<z(0)=a z^{\prime}(0)$. Since $a$ is nonnegative we have $z^{\prime}(0)>0$, which implies that $z$ is increasing to the right of $t=0$. Since $z(1)=0$ there must exist $\xi \in[0,1)$ such that $z(\xi)=\max _{t \in I} z(t)$. Then

$$
0<z(\xi), \quad 0=z^{\prime}(\xi) \quad \text { and } \quad z^{\prime \prime}(\xi) \leq 0 .
$$

The differential equation in (5) and $\left(\mathrm{H}_{\phi}\right)$ imply

$$
0 \geq z^{\prime \prime}(\xi) z(\xi)=-\left(\phi\left(\xi, x^{\prime}(\xi)\right)-\phi\left(\xi, u^{\prime}(\xi)\right)\right)\left(x^{\prime}(\xi)-u^{\prime}(\xi)\right)>0 .
$$

This is a contradiction. Similarly, if we consider the case $z(0)<0$ we will arrive at a contradiction. Hence $z(0)=0$. Now, we have a function $z$ continuous on $I$ with $z(0)=z(1)=0$. Then there exists $\tau \in I$ such that

$$
z(\tau)=\max _{t \in I} z(t), \quad z^{\prime}(\tau)=0 \quad \text { and } \quad z^{\prime \prime}(\tau) \leq 0
$$

Proceeding as before we show that $z(\tau)=0$. So that $z(t)=0$ for all $t \in I$. This shows that $x^{\prime}(t)=u^{\prime}(t)$ for all $t \in I$. Since $x(0)=u(0)=0$ it follows that $x(t)=u(t)$ for all $t \in I$, which shows the uniqueness of the solution.

Existence. For $\lambda \in[0,1]$ consider the family of problems

$$
\left\{\begin{array}{l}
-u^{\prime \prime \prime}(t)=\lambda \phi\left(t, u^{\prime}(t)\right), \quad t \in I, \\
u^{\prime}(0)-a u^{\prime \prime}(0)=\lambda \delta, \\
u^{\prime}(1)=\lambda \rho .
\end{array}\right.
$$

For $\lambda=0$ problem (6) has only the trivial solution. Thus, we consider the case $\lambda \in(0,1]$.

(i) $u$ is a solution of (6) if and only if it satisfies, for all $t \in I$,

$$
\begin{aligned}
u(t)= & \frac{\lambda t}{a+1}\left(\delta+a \rho+a \int_{0}^{1}(1-s) \phi\left(s, u^{\prime}(s)\right) d s\right) \\
& +\frac{\lambda t^{2}}{2(a+1)}\left(\rho-\delta+\int_{0}^{1}(1-s) \phi\left(s, u^{\prime}(s)\right) d s\right) \\
& -\lambda \int_{0}^{t} \frac{(t-s)^{2}}{2} \phi\left(s, u^{\prime}(s)\right) d s .
\end{aligned}
$$


Indeed, it is clear that the differential equation in (6) implies

$$
u(t)=u^{\prime}(0) t+u^{\prime \prime}(0) \frac{t^{2}}{2}-\lambda \int_{0}^{t} \frac{(t-s)^{2}}{2} \phi\left(s, u^{\prime}(s)\right) d s
$$

Then

$$
u^{\prime}(t)=u^{\prime}(0)+u^{\prime \prime}(0) t-\lambda \int_{0}^{t}(t-s) \phi\left(s, u^{\prime}(s)\right) d s .
$$

It follows that

$$
\lambda \rho=u^{\prime}(1)=u^{\prime}(0)+u^{\prime \prime}(0)-\lambda \int_{0}^{1}(1-s) \phi\left(s, u^{\prime}(s)\right) d s .
$$

But $u^{\prime}(0)=a u^{\prime \prime}(0)+\lambda \delta$, so that

$$
u^{\prime \prime}(0)=\frac{\lambda}{a+1}\left[\rho-\delta+\int_{0}^{1}(1-s) \phi\left(s, u^{\prime}(s)\right) d s\right],
$$

and consequently

$$
u^{\prime}(0)=\frac{\lambda}{a+1}\left[\delta+a \rho+a \int_{0}^{1}(1-s) \phi\left(s, u^{\prime}(s)\right) d s\right] .
$$

Now, substitute the expressions of $u^{\prime}(0)$ and $u^{\prime \prime}(0)$ into (8) to get (7).

(ii) We show that there exists a positive constant $L_{0}$, independent of $\lambda$, such that any possible solution $u$ of (6) satisfies

$$
\|u\|_{D} \leq L_{0}
$$

The boundedness of $\phi$ implies that there exists $M_{\phi}>0$ such that $\left|\phi\left(t, u^{\prime}(t)\right)\right| \leq M_{\phi}$ for all $t \in I$, so that $\left\|u^{\prime \prime \prime}\right\|_{0} \leq M_{\phi}$. Then

$$
\left|u^{\prime}(0)\right| \leq \frac{1}{a+1}\left[|\delta|+a|\rho|+a \frac{M_{\phi}}{2}\right]
$$

and

$$
\left|u^{\prime \prime}(0)\right| \leq \frac{1}{a+1}\left[|\delta|+|\rho|+\frac{M_{\phi}}{2}\right]
$$

Combining relations (9), (11), and (12) we see that

$$
\left\|u^{\prime}\right\|_{0} \leq M_{2}:=\frac{1}{a+1}\left[2|\delta|+(a+1)|\rho|+(a+1) M_{\phi}\right]
$$

Since $u(t)=\int_{0}^{t} u^{\prime}(s) d s$ it follows that

$$
\|u\|_{0} \leq M_{2}
$$


Also, $\left\|u^{\prime \prime \prime}\right\|_{0} \leq M_{\phi}$ and (12) imply

$$
\left\|u^{\prime \prime}\right\|_{0} \leq M_{1}:=\frac{1}{a+1}\left[|\delta|+|\rho|+\frac{(2 a+3) M_{\phi}}{2}\right] .
$$

Let $L_{0}=M_{1}+2 M_{2}+M_{\phi}$. Then any possible solution $u$ of (6) satisfies (10).

(iii) Define an operator $\Psi: D_{0} \rightarrow D_{0}$ by $(\Psi u)(t)=$ the right-hand side of (7). Let $\Omega:=\left\{u \in D_{0} ;\|u\|_{D} \leq L_{0}\right\}$. Then it is easily seen that $(\Psi(\Omega))$ is uniformly bounded and equicontinuous. Ascoli-Arzela theorem implies that the operator $\Psi$ is compact. Moreover, the set of all solutions $u$ of the equation $u=\lambda \Psi u$ is bounded (see (10)). It follows from Schaefer theorem (see [10]) that $u=\Psi u$ has at least one solution. Thus, (6) has at least one solution for $\lambda=1$, which is, in fact, unique from the previous step. Thus, $u$ is a solution of (5). This completes the proof of the theorem.

Remark 3 We should emphasize that, unlike Theorem 6 in [9], our Theorem 1 gives the uniqueness of the solution and this is essentially utilized in the proof of our Theorem 2 below.

For our second main result we introduce the notion of lower and upper solutions of problem (1), (2), (3), (4).

Definition 5 (a) We say that $\alpha \in D_{0}$ is a lower solution of problem (1), (3), (4) if

$$
\left\{\begin{array}{l}
-\alpha^{\prime \prime \prime}(t) \leq f\left(t, \alpha(t), \alpha^{\prime}(t), \alpha^{\prime \prime}(t)\right) \quad \text { for all } t \in I \\
\alpha^{\prime}(0)-a \alpha^{\prime \prime}(0) \leq \int_{0}^{1} h_{1}\left(\alpha(s), \alpha^{\prime}(s)\right) d s \\
\alpha^{\prime}(1) \leq \int_{0}^{1} h_{2}\left(\alpha(s), \alpha^{\prime}(s)\right) d s
\end{array}\right.
$$

(b) We say that $\beta \in D_{0}$ is an upper solution of problem (1), (3), (4) if

$$
\left\{\begin{array}{l}
-\beta^{\prime \prime \prime}(t) \geq f\left(t, \beta(t), \beta^{\prime}(t), \beta^{\prime \prime}(t)\right) \quad \text { for all } t \in I \\
\beta^{\prime}(0)-a \beta^{\prime \prime}(0) \geq \int_{0}^{1} h_{1}\left(\beta(s), \beta^{\prime}(s)\right) d s \\
\beta^{\prime}(1) \geq \int_{0}^{1} h_{2}\left(\beta(s), \beta^{\prime}(s)\right) d s .
\end{array}\right.
$$

To state and prove our second main result we introduce the following assumptions.

(A $\left.\mathrm{A}_{f}\right): I \times \mathbb{R}^{3} \rightarrow \mathbb{R}$ is continuous and satisfies

(1) there exists $C_{0}>0$ such that any solution $u$ of (1), with $u \in S(\alpha, \beta)$, satisfies $\left|u^{\prime \prime}(t)\right| \leq C_{0}$, for all $t \in I$

(2) $\left(f\left(t, u(t), v_{2}, w\right)-f\left(t, u(t), v_{1}, w\right)\right)\left(v_{2}-v_{1}\right)<0$ for $v_{1}, v_{2} \in \mathbb{R}$ such that $v_{1} \leq v_{2}$, $u \in D \cap[\alpha, \beta], w \in \mathbb{R}$ and $t \in I$

(3) $f\left(t, \alpha(t), v(t), \alpha^{\prime \prime}(t)\right) \leq f(t, u(t), v(t), w) \leq f\left(t, \beta(t), v(t), \beta^{\prime \prime}(t)\right)$ for all $u \in D \cap[\alpha, \beta]$, $v \in C^{2} \cap\left[\alpha^{\prime}, \beta^{\prime}\right], w \in \mathbb{R}$, and $t \in I$.

$\left(\mathrm{A}_{h}\right) h_{1}, h_{2}: \mathbb{R}^{2} \rightarrow \mathbb{R}$ are continuous and nondecreasing with respect to both arguments.

Remark 4 There are several sufficient conditions that imply $\left(\mathrm{A}_{f}\right)(1)$. See for instance $[6$, Lemma 1], [3, Lemma 1].

Theorem 2 Let $\alpha, \beta \in D_{0}$ be, respectively, a lower and an upper solution of problem (1), (3), (4) such that $\alpha^{\prime} \leq \beta^{\prime}$ on I. Assume that the conditions $\left(\mathrm{A}_{f}\right)$ and $\left(\mathrm{A}_{h}\right)$ are satisfied for the 
pair $(\alpha, \beta)$, where $\alpha$ is a given lower solution and $\beta$ is a given upper solution. Then problem (1), (3), (4) has at least one solution $u \in S(\alpha, \beta)$.

Proof We modify problem (1), (2), (3), (4) as follows. We define two functions $f_{C_{0}}, F: I \times$ $\mathbb{R}^{3} \rightarrow \mathbb{R}$ by

$$
f_{C_{0}}(t, u, v, w)= \begin{cases}f\left(t, p(u), v, C_{0}\right), & w \geq C_{0} \\ f(t, p(u), v, w), & |w| \leq C_{0} \\ f\left(t, p(u), v,-C_{0}\right), & w \leq-C_{0}\end{cases}
$$

and

$$
F(t, u, v, w)=f_{C_{0}}(t, u, q(v), w)= \begin{cases}f_{C_{0}}\left(t, u, \alpha^{\prime}, w\right), & v<\alpha^{\prime} \\ f_{C_{0}}(t, u, v, w), & \alpha^{\prime} \leq v \leq \beta^{\prime} \\ f_{C_{0}}\left(t, u, \beta^{\prime}, w\right), & v>\beta^{\prime}\end{cases}
$$

where $C_{0}$ is the constant from condition $\left(\mathrm{A}_{f}\right)(1)$. Consider the modified problem

$$
\left\{\begin{array}{l}
-u^{\prime \prime \prime}(t)=F\left(t, u(t), u^{\prime}(t), u^{\prime \prime}(t)\right) \quad \text { for } t \in I \\
u(0)=0 \\
u^{\prime}(0)-a u^{\prime \prime}(0)=\int_{0}^{1} h_{1}\left(u(s), u^{\prime}(s)\right) d s \\
u^{\prime}(1)=\int_{0}^{1} h_{2}\left(u(s), u^{\prime}(s)\right) d s
\end{array}\right.
$$

Define a sequence $\left(u_{j}\right)_{j \in \mathbb{N}}$ of functions in $D_{0}$ as follows. Let

$$
u_{0}(t)=\gamma t+\beta(t), \quad t \in I
$$

where $\gamma=\max _{t \in I}\left(\alpha^{\prime}-\beta^{\prime}\right)(t)$, and for $j \geq 1$,

$$
\left\{\begin{array}{l}
-u_{j}^{\prime \prime \prime}(t)=F\left(t, u_{j-1}(t), u_{j}^{\prime}(t), u_{j-1}^{\prime \prime}(t)\right) \quad \text { for } t \in I, \\
u_{j}(0)=0 \\
u_{j}^{\prime}(0)-a u_{j}^{\prime \prime}(0)=\int_{0}^{1} h_{1}\left(u_{j-1}(s), u_{j-1}^{\prime}(s)\right) d s, \\
u_{j}^{\prime}(1)=\int_{0}^{1} h_{2}\left(u_{j-1}(s), u_{j-1}^{\prime}(s)\right) d s .
\end{array}\right.
$$

We shall show that the sequence of modified problems (17) is such that each problem has a unique solution, which is uniformly bounded, together with its first and second order derivatives. Then we rely on Bolzano-Weierstrass theorem to extract a uniformly convergent subsequence, whose limit is the solution of our original problem.

1. The sequence $\left(u_{j}\right)_{j \in \mathbb{N}}$ is well defined. Indeed, for any $t \in I$ and any $z \in \mathbb{R}$ we have $q(z) \in\left[\alpha^{\prime}, \beta^{\prime}\right]$ and $p\left(u_{j-1}(t)\right) \in[\alpha, \beta]$. It follows that the function $\phi: I \times \mathbb{R} \rightarrow \mathbb{R}$, defined by

$$
\phi(t, z)=F\left(t, u_{j-1}(t), z, u_{j-1}^{\prime \prime}(t)\right)=f\left(t, p\left(u_{j-1}\right)(t), q(z), u_{j-1}^{\prime \prime}(t)\right)
$$

is continuous and bounded for all $t \in I$ and $z \in \mathbb{R}$. Moreover, condition $\left(\mathrm{A}_{f}\right)(2)$ shows that $\phi$ satisfies condition $\left(\mathrm{H}_{\phi}\right)$ in Theorem 1 . It follows from this theorem that (17) has a unique solution $u_{j}$, for each $j=1,2, \ldots$.

2. For each $j=0,1, \ldots$ the functions $u_{j}$ satisfy $u_{j} \in S(\alpha, \beta)$ and the sequence $\left(u_{j}^{\prime \prime}\right)_{j \in \mathbb{N}}$ is uniformly bounded. 
It is clear that $u_{j} \in D_{0}$ for $j=0,1, \ldots$. Since $\alpha^{\prime} \leq \beta^{\prime}$ it follows that $\gamma \leq 0$, so that $u_{0}^{\prime}=$ $\gamma+\beta^{\prime} \leq \beta^{\prime}$. On the other hand, we have $\gamma \geq-\min _{t \in I}\left(\beta^{\prime}-\alpha^{\prime}\right)(t) \geq-\beta^{\prime}(t)+\alpha^{\prime}(t)$; so that $u_{0}^{\prime} \geq \alpha^{\prime}$. It follows that $u_{0}^{\prime} \in\left[\alpha^{\prime}, \beta^{\prime}\right]$, and consequently $u_{0} \in S(\alpha, \beta)$. Also, since $u_{0}^{\prime \prime}=\beta^{\prime \prime}$ then $\left\|u_{0}^{\prime \prime}\right\|_{0} \leq\left\|\beta^{\prime \prime}\right\|_{0}$. Suppose, by induction, that we have $u_{\ell-1} \in S(\alpha, \beta)$ and there exists $K_{1} \leq C_{0}$ such that $\left\|u_{\ell-1}^{\prime \prime}\right\|_{0} \leq K_{1}$. Let

$$
\begin{aligned}
& M_{f}:=\max \left\{|f(t, u, v, w)| ; t \in I, u \in[\alpha, \beta], v \in\left[\alpha^{\prime}, \beta^{\prime}\right],|w| \leq C_{0}\right\}, \\
& \bar{h}=\max \left\{\int_{0}^{1}\left|h_{2}\left(u(s), u^{\prime}(s)\right)-h_{1}\left(u(s), u^{\prime}(s)\right)\right| d s ; u \in S(\alpha, \beta)\right\} .
\end{aligned}
$$

Claim 1 . There exists $K$ depending only on $K_{1}, M_{f}, \bar{h},\left\|\alpha^{\prime \prime}\right\|_{0}$, and $\left\|\beta^{\prime \prime}\right\|_{0}$ such that $\left\|u_{\ell}^{\prime \prime}\right\|_{0} \leq$ $K$ and $u_{\ell} \in S(\alpha, \beta)$.

To prove the claim we start with

$$
\begin{aligned}
u_{\ell}^{\prime \prime}(t) & =u_{\ell}^{\prime \prime}(0)-\int_{0}^{t} F\left(s, u_{\ell-1}(s), u_{\ell}^{\prime}(s), u_{\ell-1}^{\prime \prime}(s)\right) d s \\
& =u_{\ell}^{\prime \prime}(0)-\int_{0}^{t} f\left(s, p\left(u_{\ell-1}(s)\right), q\left(u_{\ell}^{\prime}(s)\right), u_{\ell-1}^{\prime \prime}(s)\right) d s,
\end{aligned}
$$

which leads to

$$
\left|u_{\ell}^{\prime \prime}(t)\right| \leq\left|u_{\ell}^{\prime \prime}(0)\right|+M_{f}
$$

The boundary conditions imply

$$
u_{\ell}^{\prime}(1)-u_{\ell}^{\prime}(0)=-a u_{\ell}^{\prime \prime}(0)+\int_{0}^{1}\left(h_{2}\left(u_{\ell-1}(s), u_{\ell-1}^{\prime}(s)\right)-h_{1}\left(u_{\ell-1}(s), u_{\ell-1}^{\prime}(s)\right)\right) d s
$$

On the other hand

$$
u_{\ell}^{\prime}(1)-u_{\ell}^{\prime}(0)=\int_{0}^{1} u_{\ell}^{\prime \prime}(s) d s=u_{\ell}^{\prime \prime}(0)-\int_{0}^{1}(1-s) f\left(s, p\left(u_{\ell-1}(s)\right), q\left(u_{\ell}^{\prime}(s)\right), u_{\ell-1}^{\prime \prime}(s)\right) d s
$$

It is readily seen that

$$
(a+1)\left|u_{\ell}^{\prime \prime}(0)\right| \leq \frac{M_{f}}{2}+\bar{h}
$$

Since $\left|u_{\ell-1}^{\prime \prime}(1)\right| \leq K_{1}$ (by the induction hypothesis) it follows that

$$
\left|u_{\ell}^{\prime \prime}(0)\right| \leq \frac{1}{a+1}\left(\frac{M_{f}}{2}+\bar{h}\right)=C_{1}
$$

It follows from (18) and (19) that

$$
\left|u_{\ell}^{\prime \prime}(t)\right| \leq K:=\max \left(C_{1}+M_{f},\left\|\alpha^{\prime \prime}\right\|_{0},\left\|\beta^{\prime \prime}\right\|_{0}\right) \quad \text { for } t \in I
$$

Claim 2. $u_{\ell} \in S(\alpha, \beta)$. Since $u_{\ell}(0)=0$ it suffices to show that $u_{\ell}^{\prime} \in\left[\alpha^{\prime}, \beta^{\prime}\right]$, i.e. $\alpha^{\prime} \leq u_{\ell}^{\prime} \leq \beta^{\prime}$. We, first, prove that $\alpha^{\prime} \leq u_{\ell}^{\prime}$. For this purpose, set $W(t)=u_{\ell}^{\prime}(t)-\alpha^{\prime}(t)$, for $t \in I$. We show 
that $W(t) \geq 0$ for all $t \in I$. Suppose by contradiction, that there exists $\xi_{1} \in I$ such that $W\left(\xi_{1}\right)<0$. Since $W$ is continuous, it follows that there exists $\eta \in I$ such that $W(\eta)=$ $\min \{W(t) ; t \in I\}<0$. Hence we have $W(\eta)<0, W^{\prime}(\eta)=0$ and $W^{\prime \prime}(\eta)>0$. Thus,

$$
\begin{aligned}
W^{\prime \prime}(\eta) & =u_{\ell}^{\prime \prime \prime}(\eta)-\alpha^{\prime \prime \prime}(\eta)=-F\left(\eta, u_{\ell}(\eta), u_{\ell-1}^{\prime}(\eta), u_{\ell}^{\prime \prime}(\eta)\right)-\alpha^{\prime \prime \prime}(\eta) \\
& =-f\left(\eta, p\left(u_{\ell-1}(\eta)\right), q\left(u_{\ell}^{\prime}(\eta)\right), u_{\ell-1}^{\prime \prime}(\eta)\right)-\alpha^{\prime \prime \prime}(\eta) .
\end{aligned}
$$

But $W(\eta)=u_{\ell}^{\prime}(\eta)-\alpha^{\prime}(\eta)<0$ and $W^{\prime}(\eta)=u_{\ell}^{\prime \prime}(\eta)-\alpha^{\prime \prime}(\eta)=0$. Therefore, $q\left(u_{\ell}^{\prime}(\eta)\right)=\alpha^{\prime}(\eta)$ and $u_{\ell}^{\prime \prime}(\eta)=\alpha^{\prime \prime}(\eta)$. Hence,

$$
\begin{aligned}
W^{\prime \prime}(\eta) & =-f\left(\eta,\left(p\left(u_{\ell-1}\right)\right)(\eta), q\left(u_{\ell}^{\prime}(\eta)\right), u_{\ell-1}^{\prime \prime}(\eta)\right)-\alpha^{\prime \prime \prime}(\eta) \\
& =-f\left(\eta, u_{\ell-1}(\eta), \alpha^{\prime}(\eta), u_{\ell-1}^{\prime \prime}(\eta)\right)-\alpha^{\prime \prime \prime}(\eta)>0
\end{aligned}
$$

It follows that

$$
f\left(\eta, u_{\ell-1}(\eta), \alpha^{\prime}(\eta), u_{\ell-1}^{\prime \prime}(\eta)\right)+\alpha^{\prime \prime \prime}(\eta)<0 .
$$

Since

$$
\alpha^{\prime \prime \prime}(\eta) \geq-f\left(\eta, \alpha(\eta), \alpha^{\prime}(\eta), \alpha^{\prime \prime}(\eta)\right),
$$

we infer that

$$
f\left(\eta, u_{\ell-1}(\eta), \alpha^{\prime}(\eta), u_{\ell-1}^{\prime \prime}(\eta)\right)-f\left(\eta, \alpha(\eta), \alpha^{\prime}(\eta), \alpha^{\prime \prime}(\eta)\right)<0 .
$$

The above inequality is not possible by $\left(\mathrm{A}_{f}\right)(3)$. Now, if $\eta=0$, then $W(0)<0, W^{\prime}(0) \geq 0$, and $W^{\prime \prime}(0) \geq 0$. It follows that

$$
\begin{aligned}
W(0) & =u_{\ell}^{\prime}(0)-\alpha^{\prime}(0) \\
& =a u_{\ell}^{\prime \prime}(0)+\int_{0}^{1} h_{1}\left(u_{\ell-1}(s), u_{\ell-1}^{\prime}(s)\right) d s-\alpha^{\prime}(0)<0 .
\end{aligned}
$$

Since

$$
W^{\prime}(0)=u_{\ell}^{\prime \prime}(0)-\alpha^{\prime \prime}(0) \geq 0,
$$

we get

$$
a \alpha^{\prime \prime}(0)-\alpha^{\prime}(0)+\int_{0}^{1} h_{1}\left(u_{\ell-1}(s), u_{\ell-1}^{\prime}(s)\right) d s<0 .
$$

The monotonicity of $h_{1}$ leads to

$$
a \alpha^{\prime \prime}(0)-\alpha^{\prime}(0)+\int_{0}^{1} h_{1}\left(\alpha(s), \alpha^{\prime}(s)\right) d s<0 .
$$


This is not possible by the properties of the lower solution $\alpha$. Finally, $W(1) \geq 0$. Indeed,

$$
\begin{aligned}
W(1) & =u_{\ell}^{\prime}(1)-\alpha^{\prime}(1)=\int_{0}^{1} h_{2}\left(u_{\ell-1}(s), u_{\ell-1}^{\prime}(s)\right) d s-\alpha^{\prime}(1) \\
& \geq \int_{0}^{1} h_{2}\left(\alpha(s), \alpha^{\prime}(s)\right) d s-\alpha^{\prime}(1) \geq 0
\end{aligned}
$$

by definition of the lower solution $\alpha$. Similarly we show that $u_{\ell}^{\prime} \leq \beta^{\prime}$. Thus, we have shown that $u_{\ell}^{\prime} \in\left[\alpha^{\prime}, \beta^{\prime}\right]$, which implies that $u_{\ell} \in S(\alpha, \beta)$. Therefore, we have proved that the sequences $\left(u_{j}^{\prime \prime}\right)_{j \in \mathbb{N}},\left(u_{j}^{\prime}\right)_{j \in \mathbb{N}}$, and $\left(u_{j}\right)_{j \in \mathbb{N}}$ are uniformly bounded on the interval $I$. BolzanoWeierstrass theorem implies that we can extract subsequences $\left(u_{j_{m}}^{\prime \prime}\right)_{j_{m} \in \mathbb{N}},\left(u_{j_{m}}^{\prime}\right)_{j_{m} \in \mathbb{N}}$ and $\left(u_{j_{m}}\right)_{j_{m} \in \mathbb{N}}$ that are uniformly convergent on $I$. Using the diagonalization process, if necessary, we shall assume that $\lim _{m \rightarrow \infty} u_{j_{m}}^{\prime \prime}=\lim _{m \rightarrow \infty} u_{j_{m-1}}^{\prime \prime}=w, \lim _{m \rightarrow \infty} u_{j_{m}}^{\prime}=\lim _{m \rightarrow \infty} u_{j_{m-1}}^{\prime}=$ $v$ and $\lim _{m \rightarrow \infty} u_{j_{m}}=\lim _{m \rightarrow \infty} u_{j_{m-1}}=u$. To complete the proof of our second main result we prove that $u^{\prime}=v, u^{\prime \prime}=w$ and $u$ is the desired solution of our original problem. Since $u_{j_{m}}(t)=\int_{0}^{t} u_{j_{m}}^{\prime}(s) d s$, it follows from the uniform convergence of the two subsequences that $u(t)=\int_{0}^{t} v(s) d s$, and this equality implies that $u(0)=0$ and $u^{\prime}=v$. Also, we have $u_{j_{m}}(t)=u_{j_{m}}(0)+u_{j_{m}}^{\prime}(0) t+\int_{0}^{t}(t-s) u_{j_{m}}^{\prime \prime}(s) d s=u_{j_{m}}^{\prime}(0) t+\int_{0}^{t}(t-s) u_{j_{m}}^{\prime \prime}(s) d s$, which implies that $u(t)=u^{\prime}(0) t+\int_{0}^{t}(t-s) w(s) d s$, from which we readily get $u^{\prime \prime}=w$. It is clear that

$$
u \in S(\alpha, \beta) \text { and }\left\|u^{\prime \prime}\right\|_{0} \leq K
$$

The differential equation $-u_{j_{m}}^{\prime \prime \prime}(t)=F\left(t, u_{j_{m}-1}(t), u_{j_{m}}^{\prime}(t), u_{j_{m}-1}^{\prime \prime}(t)\right)$, for $t \in I$, implies that $-u_{j_{m}}^{\prime \prime}(t)=-u_{j_{m}}^{\prime \prime}(0)+\int_{0}^{t} F\left(s, u_{j_{m}-1}(s), u_{j_{m}}^{\prime}(s), u_{j_{m}-1}^{\prime \prime}(s)\right) d s$. The continuity of $F$ and the uniform convergence of the respective subsequences imply that $-u^{\prime \prime}(t)=-u^{\prime \prime}(0)+\int_{0}^{t} F\left(s, u(s), u^{\prime}(s)\right.$, $\left.u^{\prime \prime}(s)\right) d s$, so that

$$
-u^{\prime \prime \prime}(t)=F\left(t, u(t), u^{\prime}(t), u^{\prime \prime}(t)\right) \quad \text { for } t \in I \text {. }
$$

The definition of $F$ and (21) show that

$$
-u^{\prime \prime \prime}(t)=f\left(t, u(t), u^{\prime}(t), u^{\prime \prime}(t)\right) \quad \text { for } t \in I \text {. }
$$

Similarly we can show that

$$
u^{\prime}(0)-a u^{\prime \prime}(0)=\int_{0}^{1} h_{1}\left(u(s), u^{\prime}(s)\right) d s
$$

and

$$
u^{\prime}(1)=\int_{0}^{1} h_{2}\left(u(s), u^{\prime}(s)\right) d s
$$

We see that $u$ is a solution of (1), (2), (3), (4). Moreover, $u \in S(\alpha, \beta)$. This completes the proof of our main result. 
Authors' contributions

All authors contributed equally to the writing of this paper. All authors read and approved the final manuscript.

\section{Author details}

'Department of Mathematics and Statistics, King Fahd University of Petroleum and Minerals, P.O. Box 5046, Dhahran, 31261, Saudi Arabia. ${ }^{2}$ Department of Mathematics, University of Tlemcen, P.O. Box 119, Tlemcen, 13000, Algeria.

${ }^{3}$ Department of Mathematics, Dammam University, P.O. Box 838, Dammam, Saudi Arabia.

\section{Acknowledgements}

A Boucherif is grateful to King Fahd University of Petroleum and Minerals for its constant support. The authors are grateful to the anonymous referees and the handling editor for comments that led to the improvement of the presentation of the manuscript.

Received: 23 January 2014 Accepted: 23 May 2014 Published online: 10 September 2014

\section{References}

1. Boucherif, A, Al-Malki, N: Nonlinear three-point third order boundary value problems. Appl. Math. Comput. 190, 1168-1177 (2007)

2. Minhós, FM: On some third order nonlinear boundary value problems: existence, location and multiplicity results. J. Math. Anal. Appl. 339, 1342-1353 (2008)

3. Grossinho, MR, Minhós, FM: Existence results for some third order separated boundary value problems. Nonlinear Anal. 47, 2407-2418 (2001)

4. Klokov, YA: Upper and lower functions in boundary value problems for third-order ordinary differential equations. Differ. Equ. 36, 1762-1769 (2000)

5. Boucherif, A, Bouguima, SM, Al-Malki, N, Benbouziane, ZN: Third order differential equations with integral boundary conditions. Nonlinear Anal. 71, e1736-e1743 (2009)

6. Benbouziane, ZN, Boucherif, A, Bouguima, SM: Third order nonlocal multipoint boundary value problems. Dyn. Syst. Appl. 13, 41-48 (2004)

7. Gray, M: Uniqueness implies uniqueness for nonlocal boundary value problems for third order ordinary differential equations. Dyn. Syst. Appl. 16, 277-284 (2007)

8. Wang, Y, Ge, W: Existence of solutions for a third order differential equation with integral boundary conditions. Comput. Math. Appl. 53, 144-154 (2007)

9. Graef, J, Kong, L, Minhós, F: Higher order functional boundary value problems: existence and location results. Acta Sci. Math. 77, 87-100 (2011)

10. Smart, DR: Fixed Point Theorems. Cambridge University Press, Cambridge (1974)

doi:10.1186/s13661-014-0137-z

Cite this article as: Boucherif et al.: Third order problems with nonlocal conditions of integral type. Boundary Value Problems 2014 2014:137.

\section{Submit your manuscript to a SpringerOpen ${ }^{\circ}$ journal and benefit from:}

- Convenient online submission

Rigorous peer review

- Immediate publication on acceptance

- Open access: articles freely available online

- High visibility within the field

- Retaining the copyright to your article 